\title{
Localization of Changes in a Model Winding Based on Terminal Measurements: Experimental Study
}

\author{
K. Ragavan and L. Satish, Senior Member, IEEE
}

\begin{abstract}
By using some properties of driving-point functions and adopting an iterative circuit synthesis approach, the location, extent, and type of change introduced in a model winding could be identified, based on terminal measurements. In this study, a model winding was used. From knowledge of its measured shortcircuit and open-circuit natural frequencies, and pertinent winding data, an equivalent circuit was synthesized (called reference circuit). Next, changes were introduced at different locations in the model winding and its natural frequencies were measured. Corresponding to every new set of measured natural frequencies, a new circuit was synthesized (with topology remaining unchanged). A comparison of these circuits with the reference circuit revealed that a mapping could be established between changes introduced in the model winding and those predicted by the synthesized circuits. Many case studies are presented by considering continuous-disc and interleaved winding representations. Reasonably good results were obtained. Thus, localization of changes, based on terminal measurements, is shown to be a possibility. So, it is believed that these findings could be of some assistance in addressing the ultimate task of locating mechanical deformations in actual transformer windings.
\end{abstract}

Index Terms-Circuit synthesis, driving-point functions, opencircuit and short-circuit natural frequencies, winding deformation and localization.

\section{INTRODUCTION}

$\mathbf{O}$ VER THE YEARS, condition monitoring and diagnostic methods, such as low-voltage impulse testing and frequency response or the transfer function method, have been shown to possess abilities to detect mechanical deformations of transformer windings [1]-[4]. In this context, literature reveals that diagnosis (which by definition means interpretation of monitored data to draw inferences), has at the most been confined to a mere comparison of two subsequently acquired data sets. During such a comparison, if a deviation is detected, a possible winding damage is inferred. When confronted with such a situation, it is natural to anticipate questions like the following.

1) What is the extent of the deformation?

2) Where along the winding are they located?

Ideally, the diagnosis tool is expected to be endowed with powers to answer these questions. Although much has been written about their diagnostic capabilities, the fact remains that these answers are still elusive. It is needless to say that finding

Manuscript received March 17, 2006; revised June 22, 2006. Paper no. TPWRD-00141-2006.

The authors are with the Indian Institute of Science, Bangalore 560012, India (e-mail: satish@hve.iisc.ernet.in).

Digital Object Identifier 10.1109/TPWRD.2006.886789

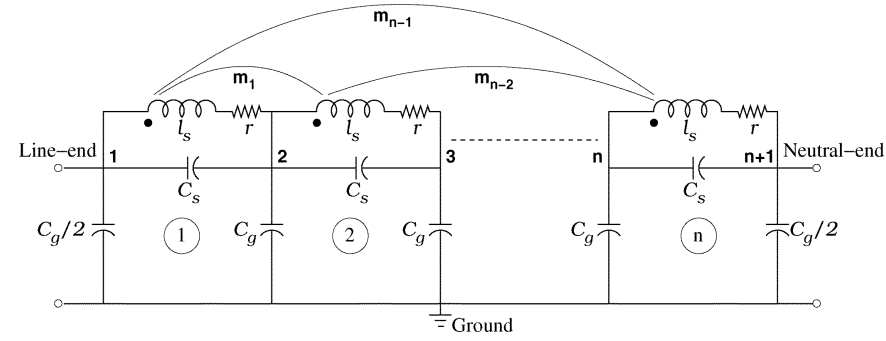

Fig. 1. Equivalent circuit of a transformer winding.

these answers is crucial. Therefore, it is desirable to explore alternative approaches possessing these capabilities. This is the motivation.

\section{Problem Definition ANd Underlying PRinciPle}

As this was a preliminary effort, wherein the proposed method was being evolved, it was deemed appropriate to consider only a model winding. This choice is in fact advantageous due to the following:

1) convenience of introducing any change (increase and/or decrease);

2) more important, the quantum of change introduced is precisely known;

3) permits objective evaluation of proposed approach.

Quite clearly, achieving some of the above with an actual transformer winding is cumbersome. With this in mind, the objective of this paper can be stated as- Based on input terminal measurements, propose a method to locate the position, as well as, identify the extent of changes introduced in the model winding.

In order to achieve this goal of localization, it appeared worthwhile to convert the model winding (via circuit synthesis) into an equivalent circuit (with suitable number of sections) as in Fig. 1. The main reason for this choice is that this representation inherently captures the physical length of the winding. Or, in other words, the physically continuous winding can be visualized as being mapped onto a series of discrete nodes, starting from line to neutral end. This feature afforded by the equivalent circuit (in addition to adequately describing the lightning-impulse behavior) was found to be ideally suited for this task.

Essentially this task turns out to be a circuit synthesis exercise, subject to the condition that the synthesized circuit should exhibit the same terminal characteristics as that of the model winding (i.e., difference between all their corresponding shortcircuit natural frequencies (scnf) and open-circuit natural frequencies (ocnf) should be within a specified tolerance). 
Circuit synthesis is, in general, a nonunique operation. This restraint was overcome by using properties exhibited by drivingpoint functions, assuming a fixed circuit topology, and adopting an iterative circuit synthesis approach. An additional circuit is synthesized corresponding to every new set of measured natural frequencies. Thus, a comparison of every synthesized circuit with the reference case reveals, which parts of the model winding have changed and also the quantum of change.

\section{LITERATURE}

Literature pertinent to the objectives of this paper were examined. It emerges that earlier efforts have basically been confined to the following areas, viz.

- Estimating circuit parameters from winding design details: Knowing physical dimensions, winding configuration and the insulation structure, the capacitances and inductances of the ladder network are estimated in [5] and [6]. However, in [7], it is shown that it is possible to calculate the turn leakage inductances and capacitances, given the design details of the transformer windings. These methods are helpful (during the design stage) to predetermine the transformer performance.

- Building circuit models using terminal measurements:

Based on terminal measurements on the winding, there have been some efforts to construct circuit models (nonladder networks) of the windings [8]-[11]. In [12], only the self and mutual inductances of a ladder network (of a prefixed size) have been estimated. Two nonladder networks were constructed [13] using frequency response data corresponding to a transformer winding before and after the introduction of a fault. Although the terminal characteristics matched, the circuits synthesized do not correspond to physically realizable networks, as the resistances involved in them are negative and the inductances are far too high. So, localization of the faults appears to be remote following these approaches.

- Rational function approximation of terminal characteristics:

Although there have been some efforts to construct a rational function approximation of terminal characteristics (in s-domain) using frequency response data [14]-[16], they were not always physically realizable. For example, if the driving-point function constructed is not a ratio of Hurwitz polynomials, then it cannot be represented by a physically realizable network [17]. Nevertheless, these functional models were useful for representing the terminal behavior of transformers in power system studies.

Thus, from the above, it emerges that the objective of localization of winding deformations based on terminal measurements, has so far not been addressed and hence forms the subject matter of this paper.

\section{Synthesize REFERENCE CIRCUIT}

The first step in the proposed method involves representing the model winding by means of an equivalent circuit, called the reference circuit. To achieve this, the following quantities of the model winding are required:

- dc resistance $R_{\mathrm{dc}}$;

- equivalent air-core inductance $L_{\text {eq }}$;

- effective shunt capacitance to ground $C_{\mathrm{g}, \text { eff }}$;

- initial voltage distribution constant $\alpha$.

Among these, the quantities $R_{\mathrm{dc}}, L_{\mathrm{eq}}$ and $C_{\mathrm{g} \text {,eff }}$ are measurable at the input terminals of the model and the initial voltage distribution constant $(\alpha)$ can be got from design details. The steps involved in synthesizing a circuit to represent the model winding are as follows.

A) Determine natural frequencies (ocnfs and scnfs) of the model winding by swept frequency measurements.

B) Determine the number of sections of the equivalent circuit to be synthesized.

C) Estimate individual values of the elements of the equivalent circuit.

Each of these steps will be discussed below.

\section{A. Determine Natural Frequencies}

The driving-point impedance function of an n-section equivalent circuit (as in Fig. 1) with its neutral grounded can be expressed as [18]

$$
Z(s)=\frac{\beta_{1}(s-\tau) \prod_{i=1}^{n-1}\left(s-z_{i}\right)\left(s-z_{i}^{*}\right)}{\prod_{i=1}^{n}\left(s-p_{i}\right)\left(s-p_{i}^{*}\right)}
$$

where

$$
\begin{array}{ll}
\beta_{1} & \text { scaling factor; } \\
\tau & \text { real zero; } \\
z_{i}, z_{i}^{*} & \text { complex conjugate zero pair; } \\
p_{i}, p_{i}^{*} & \text { complex conjugate pole pair. }
\end{array}
$$

Poles and zeros of the driving-point impedance function lie in the left half of the complex frequency plane. For driving-point functions, in the complex poles $\left(\sigma_{p}+j \omega_{p}\right)$ and complex zeros $\left(\sigma_{z}+j \omega_{z}\right)$, the real parts are due to the losses (represented as resistances) and the imaginary parts signify the angular frequencies at which the peaks and troughs occur in the magnitude plot of the driving-point function.

1) Natural Frequencies Pertaining to Second-Order Poles and Zeros: To determine the natural frequencies of the model winding, swept frequency measurements are performed on it. This essentially involves varying the excitation frequency over a wide range and at every frequency, the magnitude and phase of the input voltage and current are measured, using which the driving-point impedance is determined.

The measured magnitude and phase plots of the driving-point impedance corresponding to a continuous-disc model winding (details are described in Section VI) are presented in Fig. 2. From this figure, the following points emerge.

- At certain frequencies, the magnitude of the driving-point impedance becomes maximum and simultaneously its phase becomes zero (while changing from positive to negative). These frequencies are referred as open-circuit natural frequencies (ocnf $f_{p}$ 's). 


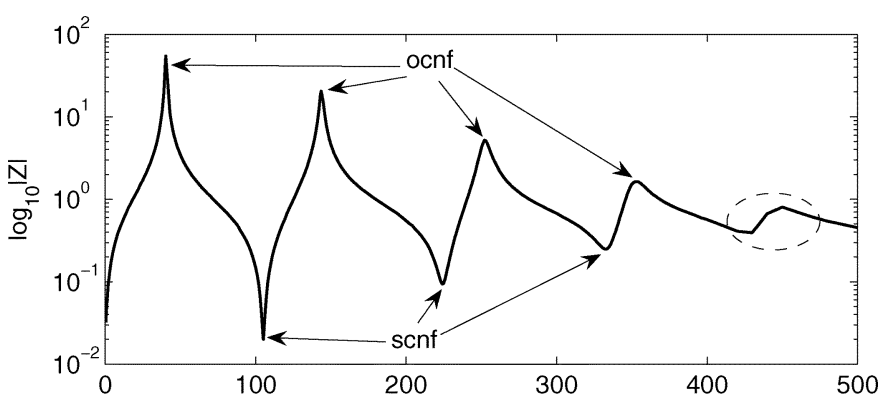

(a)

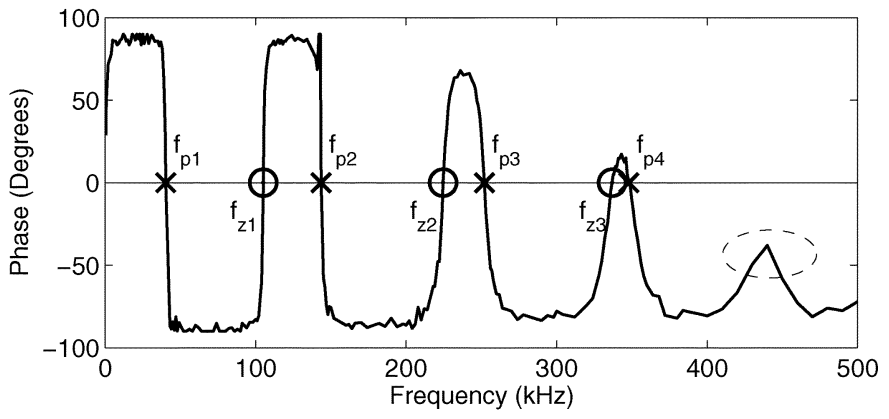

(b)

Fig. 2. Measured driving-point impedance of the model winding (a) magnitude and (b) phase.

- At some frequencies, the magnitude of the driving-point impedance becomes minimum and its phase simultaneously becomes zero (while changing from negative to positive), and these frequencies are referred as short-circuit natural frequencies ( $\operatorname{scnf} f_{z}$ 's).

Thus, ocnfs and scnfs pertaining to the second-order poles and zeros of driving-point impedance function can be determined by inspection of the magnitude and phase plots.

2) Natural Frequency (scnf) Pertaining to First-Order Zero: The driving-point impedance function (1) contains a real zero $\tau$ (where, $\tau=-2 \pi f_{1}$ ). As the loss is generally very low, the scnf ' $f_{1}$ ' would lie in the low-frequency region (of the order of a few hundreds of hertz) and it can be determined as follows. The impedance $Z(s)$ at low frequencies is

$$
\lim _{s \rightarrow 0} Z(s)=R_{\mathrm{dc}}+s L_{\mathrm{eq}} .
$$

From the above, the real zero $(s=\tau)$ is found to be

$$
\tau=-\frac{R_{\mathrm{dc}}}{L_{\mathrm{eq}}} \text {. }
$$

\section{B. Determine the Number of Sections $N$}

While synthesizing the circuit, determination of the number of sections $N$, is very crucial. For estimating it, the rule-ofthumb mentioned in [19] can be used (i.e., "the number of sections to be used in the equivalent circuit can be chosen somewhat larger than the required number of natural frequencies"). From this, at first glance it appears as though the determination of $N$ from the magnitude plot is straightforward. However, this is true, if and only if all the natural frequencies are observable. But in practice, not all of them are observable, because of the following reasons.
- At higher frequencies, due to skin-effect, damping is high. Hence, the high frequency scnfs and ocnfs are not well-pronounced in the frequency response plots (Fig. 2). Thus, the true value of $N$ (minimum number of sections the equivalent circuit should have to reproduce the same terminal characteristics as that of the winding) cannot be ascertained based on a mere count of the number of peaks and troughs.

- Corresponding to ocnf (or peaks in the magnitude plot), the current drawn from the source would approach zero. This makes it difficult to differentiate between signal and noise, in spite of adopting signal averaging techniques afforded by digital oscilloscopes. Further, it also becomes increasingly difficult to precisely identify a zero crossing of the phase angle.

- In an interleaved winding, since most of the high-frequency zeros and poles lie very close to each other, only a few natural frequencies are observable in the frequency response plots [20]. In such cases, determining the true value of $N$ based only on an inspection of the frequency-response plots is not possible.

- Further, in actual measurements, the frequency response plots exhibit a few tiny peak-like kinks. These artifacts are due to noise, limited sensitivity of the measuring instruments, etc. Hence, estimation of $N$ is fraught with difficulties.

- Sometimes, a well-defined trough/peak may occur in the magnitude plot, but the corresponding phase may not attain zero. Such frequencies will not be considered as scnf/ ocnf. One example is seen in Fig. 2, wherein the encircled trough/peak has not been considered as scnf/ocnf.

Due to the above reasons, the number of clearly identifiable peaks is always less than $N$ (i.e., $q$ peaks and $(q-1)$ troughs). Utilizing this limited information, how the value of $N$ can actually be arrived at is discussed next.

At high frequencies, the impedance offered by the circuit is found from (1) as

$$
\lim _{s \rightarrow \infty} Z(s)=\frac{\beta_{1}}{s} .
$$

Since, the circuit behaves entirely as a capacitive network at higher frequencies, the scaling factor $\beta_{1}$ can be related to the equivalent capacitance $C_{\text {eq }}$ as

$$
\beta_{1}=\frac{1}{C_{\mathrm{eq}}} .
$$

The above relation is a crucial link which can be made use for determining $N$.

At $s=0$, the input impedance becomes resistive (i.e., $R_{\mathrm{dc}}$ ). Using this and substituting (3) in (1), the scaling factor $\beta_{1}$ can be expressed as

$$
\beta_{1}=L_{\mathrm{eq}} \times \frac{\prod_{i=1}^{n}\left(-p_{i}\right)\left(-p_{i}^{*}\right)}{\prod_{i=1}^{n-1}\left(-z_{i}\right)\left(-z_{i}^{*}\right)} .
$$

Since only $q$ ocnfs and $(q-1)$ scnfs are observable, $n$ and $(n-1)$ in the above equation can be replaced by $q$ and $(q-1)$, respectively.

Also, as the absolute value of the imaginary parts of complex frequencies is significantly higher than their real parts (losses 
in the winding are low), $p_{i}$ 's and $z_{i}$ 's in (6) can be replaced by the angular frequencies $\omega_{p}$ 's and $\omega_{z}$ 's, respectively. Since imaginary parts of poles and zeros are $\omega_{p}=2 \pi f_{p}$ and $\omega_{z}=$ $2 \pi f_{z}$, respectively, (6) can be rewritten as

$$
\beta_{1} \approx L_{\mathrm{eq}} \times \frac{4 \pi^{2} \prod_{i=1}^{q} f_{p_{i}}^{2}}{\prod_{i=1}^{q-1} f_{z_{i}}^{2}}
$$

where frequencies and inductance are expressed in $\mathrm{MHz}$ and $\mathrm{mH}$, respectively.

One of the unique properties of driving-point impedance function [18] is that its poles and zeros alternate with each other. That is, every trough (complex conjugate zero pair) alternates with a peak (complex conjugate pole pair). Further, the number of peaks is more than the troughs by unity. (Note: This feature is not exhibited by the transfer function and hence instead of it the driving-point impedance function was chosen in this study.)

From Fig. 2(b), by inspection, it can be inferred that the ratio of the square of any ocnf to that of the preceding scnf is always greater than unity. Hence, if a ocnf-scnf pair (or peak-trough pair) is not accounted for (due to the reasons mentioned before), then the value of $\beta_{1}$ estimated with reduced number of ocnfs and scnfs will always be less than its actual value and, hence, (5) would become modified as

$$
\left(\frac{1}{C_{\mathrm{eq}}}-\beta_{1}\right)>0 \text {. }
$$

The above inequality forms the basis for finding $N$ and the steps involved for its estimation are given in Algorithm 1.

Algorithm 1 Determining Number of Sections

1) Measure ocnfs $\left\{f_{p_{i}}, \forall i=1, \ldots, q\right\}$ and scnfs $\left\{f_{z_{i}}, \forall i=1, \ldots, q-1\right\}$ of the model winding.

2) Determine $\beta_{1}$ using equation (7).

3) $N \leftarrow q$

4)

$$
C_{g}=\frac{C_{\mathrm{g}, \mathrm{eff}}}{N} ; C_{s}=\frac{C_{\mathrm{g}, \mathrm{eff}} \times N}{\alpha^{2}} .
$$

5) Estimate $C_{\text {eq }}$ of the ladder network with $N$ sections as

$$
C_{\mathrm{eq}}=\frac{C_{g}}{2}+\frac{1}{\frac{1}{C_{s}}+\frac{1}{C_{g}+\frac{1}{C_{s}}+\cdots}} .
$$

6) if $\left(\left(\frac{1}{C_{\mathrm{eq}}}-\beta_{1}\right)<0\right)$, then

7) $N \leftarrow N+1$.

GOTO step 4

8) end if

\section{Determine Elements of Reference Circuit}

1) Capacitances and Resistances: From the values of $C_{g}$ and $C_{s}$ (capacitances per section) determined in (9), the nodal capacitance matrix $\left[K_{\text {ref }}\right]$ can be constructed. Further, the sectional value of the resistance can be determined as

$$
r=\frac{R_{\mathrm{dc}}}{N} .
$$

Let $\left[R_{\mathrm{ref}}\right]$ be the diagonal matrix with its elements equal to $r$.

2) Inductances: Let $l_{s}$ be the self-inductance of the first section and $m_{1}, m_{2}, \ldots m_{N-1}$ be the mutual inductances between the first and the other sections in the circuit, respectively. The magnetic coupling between individual sections decreases when their separation distance increases. So it is logical to consider that the self and mutual inductances satisfy the following constraints:

$0.4 l_{s}<m_{1}<0.8 l_{s}$
$0.4 m_{i-1}<m_{i}<0.8 m_{i-1}, \forall i=2, \ldots, N-1$.

The limits, namely 0.4 and 0.8 , were found to be suitable in this work and can be changed, if needed. Assuming symmetry, the remaining self and mutual inductances can be taken to be the same. The inductance matrix $[L]$ is constructed such that its diagonal elements are equal to the values of the self inductances, and the nondiagonal elements are the mutual inductances. The estimation of mutual inductances can be accomplished by imposing (12) and following an iterative procedure as explained in Algorithm 2. (Note: In case, if the symmetry does not exist, then a similar constraint as in (12) can be chosen for the other sections also).

Algorithm 2 Determining inductances of the reference circuit

1) $\left\{l_{s}, m_{1}, m_{2}, \ldots, m_{N-1}\right\} \leftarrow$ minimum values as per equation (12).

2) Increase the value of one inductance by $1 \%$ of $l_{s}$ (either self or mutual) and estimate the equivalent inductance as

$$
L_{\text {eq }}(\text { estimated })=N l_{s}+2 \sum_{i=1}^{N-1}(N-i) m_{i} \text {. }
$$

3) if $\left(L_{\text {eq }}\right.$ (estimated $) \approx L_{\text {eq }}$, within $1 \%$ tolerance) then

4) Construct inductance matrix $[L]$.

5) Estimate ocnfs $\left\{f_{p_{i}}, \forall i=1, \ldots, N\right\}$ and scnfs $\left\{f_{z_{i}}, \forall i=1, \ldots, N-1\right\}$ using $\left[K_{\text {ref }}\right],\left[R_{\text {ref }}\right],[L]$.

6) if $\left(\operatorname{estimated}\left\{f_{p}, f_{z}\right\} \approx\right.$ measured $\left\{f_{p}, f_{z}\right\}$, within $1 \%$ tolerance) then

7) $\left[L_{\mathrm{ref}}\right]=[L]$.

8) else

9) GOTO step 2.

10) end if

11) else

12) GOTO step 2.

13) end if 
Thus, $\left[K_{\text {ref }}\right],\left[L_{\text {ref }}\right]$, and $\left[R_{\text {ref }}\right]$ correspond to the reference circuit. These will be subsequently used as an initial guess to synthesize further circuits, when changes are introduced in the model winding.

\section{Synthesize Circuit After Changes ARe Introduced}

The proposed method involves the following steps.

1) A few elements in the model winding are changed and $R_{\mathrm{dc}}$, $L_{\mathrm{eq}}$, and $C_{\mathrm{g}, \mathrm{eff}}$ are measured.

2) Swept-frequency measurement is performed and all wellpronounced ocnfs and scnfs are identified.

3) A comparison of these frequencies with those corresponding to the reference case is made. A deviation that is greater than the tolerance limit $(2 \%)$ would indicate that the model winding has undergone a change. As resistances in the circuit do not have much of an impact on the natural frequencies, it can be considered that the changes observed in the natural frequencies are only due to changes in capacitances and/or inductances.

4) Using the measured values $R_{\mathrm{dc}}, L_{\mathrm{eq}}, C_{\mathrm{g} \text {,eff }}$, ocnfs, and scnfs, an equivalent circuit is synthesized for representing the model winding after changes. For this purpose, the matrices describing the reference circuit are used as the initial guess. Its elements are iteratively varied until the estimated values of the equivalent inductance, effective shunt capacitance, and the natural frequencies satisfy the following condition (within $2 \%$ tolerance)

$$
\begin{aligned}
\text { estimated }\left\{L_{\text {eq }}, C_{\mathrm{g}, \mathrm{eff}}\right\} & \approx \operatorname{measured}\left\{L_{\mathrm{eq}}, C_{\mathrm{g}, \mathrm{eff}}\right\} \\
\text { estimated }\{\text { ocn } f, \text { scn } f\} & \approx \operatorname{measured}\{\text { ocn } f, \text { scnf }\} .
\end{aligned}
$$

Let $[\widehat{K}]$ and $[\widehat{L}]$ be the matrices (corresponding to the model winding after changes) satisfying (14). Estimation of these matrices involves a vast search space, which requires extensive computational time. Further, it depends on factors viz. size of the equivalent circuit, number of changed elements, and their position from the line end and step size used in the iterative process and the specified tolerances. A comparison of $[\widehat{K}]$ and $[\widehat{L}]$ with $\left[K_{\text {ref }}\right]$ and $\left[L_{\text {ref }}\right]$ reveals the elements that have changed. The row and column of the changed element in the matrix reveals the position along the winding where the changes have been made. Further, the extent of change and whether the change is confined to inductances and/or capacitances, can also be ascertained.

\section{EXPERIMENTAL RESULTS}

The model winding used to demonstrate the method consisted of a single-layer wound on an insulated air-core former of diameter $200 \mathrm{~mm}$. The winding had 200 turns and taps were provided after every 20 turns. Using these taps, series and shunt capacitances could be connected externally to represent continuousdisc and interleaved windings. The taps also facilitated introduction of specific changes at any location along the model winding. Representation of both continuous-disc and interleaved type of windings were considered.

\section{A. Case Studies With Continuous-Disc Windings}

Initially, a continuous-disc winding representation was chosen by connecting $C_{g}=0.56 \mathrm{nF}, C_{s}=1 \mathrm{nF}$ (refer to
TABLE I

\begin{tabular}{|c|c|c|c|c|}
\hline \multirow{2}{*}{\multicolumn{2}{|c|}{$\begin{array}{l}\text { Measured with } \\
\text { Model winding }\end{array}$}} & \multicolumn{2}{|c|}{ Estimated using } & \multirow{3}{*}{ Error $^{\dagger}$} \\
\hline & & \multicolumn{2}{|c|}{ Synthesized circuit } & \\
\hline $\begin{array}{l}\mathbf{s c n f} \\
(\mathrm{kHz})\end{array}$ & $\begin{array}{c}\text { ocnf } \\
(\mathrm{kHz})\end{array}$ & $\begin{array}{c}\text { scnf } \\
(\mathrm{kHz})\end{array}$ & $\begin{array}{l}\text { ocnf } \\
(\mathrm{kHz})\end{array}$ & \\
\hline 105 & 40.3 & 105.19 & 40.48 & \multirow{6}{*}{$<0.5 \%$} \\
\hline 223.8 & 143 & 224.79 & 142 & \\
\hline \multirow[t]{4}{*}{336} & 250.6 & 334.44 & 249.42 & \\
\hline & 346 & 410.09 & 346.41 & \\
\hline & & 472.42 & 414.34 & \\
\hline & & & 473.73 & \\
\hline \multicolumn{2}{|c|}{$L_{e q}=6.98 \mathrm{mH}$} & \multicolumn{2}{|c|}{$L_{e q}=7.0385 \mathrm{mH}$} & 0.84 \\
\hline
\end{tabular}

MEASURED AND ESTIMATED FREQUENCIES FOR REFERENCE CASE

TABLE II

ESTIMATING NUMBER OF SECTIONS $N$

\begin{tabular}{c|c|c|c}
\hline Sections $(N)$ & 4 & 5 & 6 \\
\hline$C_{e q}(\mathrm{nF})$ & 1.0247 & 0.9347 & 0.8819 \\
\hline$\frac{1}{C_{e q}}-\beta_{1}$ & $-\mathrm{ve}$ & $-\mathrm{ve}$ & $+\mathrm{ve}$ \\
\hline
\end{tabular}

Fig. 1) at the taps available in the winding, which results in $\alpha=7.4833$. Then, the following quantities viz. $R_{\mathrm{dc}}=8 \Omega$, $L_{\mathrm{eq}}=6.98 \mathrm{mH}$, and $C_{\mathrm{g}, \mathrm{eff}}=5.6 \mathrm{nF}$ were measured.

1) Constructing Reference Circuit: Swept-frequency measurements were carried out using 1) function generator, 2) digital storage oscilloscope, and 3) current probe, and the magnitude and phase of the driving-point impedance were measured and shown in Fig. 2. Although five peaks are seen in the magnitude plot Fig. 2(a), the fifth encircled trough/peak is discarded as the phase corresponding to those frequencies is not becoming zero. The well-pronounced natural frequencies (corresponding to four peaks and three troughs) were identified and are listed in Table I.

For estimating the number of sections $N$ in the circuit to be synthesized, Algorithm 1 is applied. Substituting the measured values of $L_{\mathrm{eq}}$ and the natural frequencies in (7), the scaling factor $\beta_{1}$ of the driving-point impedance function is estimated to be $\beta_{1}=1.1045$.

Since $q=4$ (number of well-pronounced peaks), initially, the equivalent capacitance of a 4 -section ladder network is determined. Its value is found to be $C_{\mathrm{eq}}=1.0247 \mathrm{nF}$ and it does not satisfy (8). Hence, the circuit to be synthesized should have $N>4$.

Then, the number of sections in the circuit are sequentially increased until the value of $C_{\mathrm{eq}}$ satisfies (8). Corresponding to $N=6$, the sectional values of capacitances turn out to be $C_{g}=0.933 \mathrm{nF}, C_{s}=0.6 \mathrm{nF}$, using which $C_{\mathrm{eq}}$ is estimated. It is seen from Table II that, the 6-section circuit satisfies (8) and, hence, the minimum number of sections in the circuit to represent the model winding should therefore be $N=6$. This must be contrasted with the four peaks observed in the magnitude plot (Fig. 2). But by using (8), it has been possible to arrive at $N=6$. Then, the resistance per section estimated as $r=1.333 \times 10^{-3} \mathrm{k} \Omega$. Using these sectional values of the capacitances and resistance, the matrices $\left[K_{\text {ref }}\right]$ and $\left[R_{\text {ref }}\right]$ can be constructed.

The self and mutual inductances are estimated iteratively as explained in Algorithm 2 and the estimated values are presented 
TABLE III

Estimated VALUES OF SELF AND Mutual InduCTANCES ${ }^{\dagger}$

\begin{tabular}{c|c|c|c|c|c}
\hline$l_{s}$ & $m_{1}$ & $m_{2}$ & $m_{3}$ & $m_{4}$ & $m_{5}$ \\
\hline 0.4310 & 0.2392 & 0.1435 & 0.0947 & 0.0612 & 0.0496 \\
\hline
\end{tabular}

values are in $\mathrm{mH}$

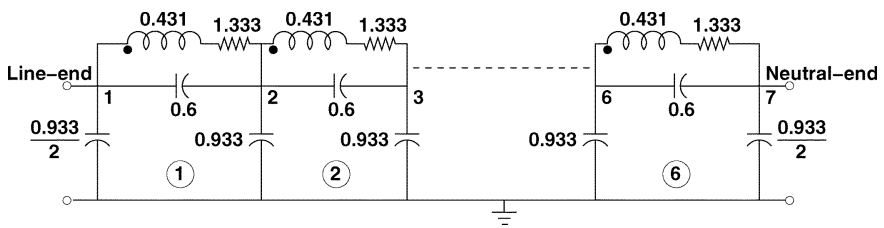

Fig. 3. Six-section synthesized reference circuit (capacitances, inductances, and resistances are in $\mathrm{nF}, \mathrm{mH}$, and $\Omega$, respectively, and refer to Table III for mutual inductance values)

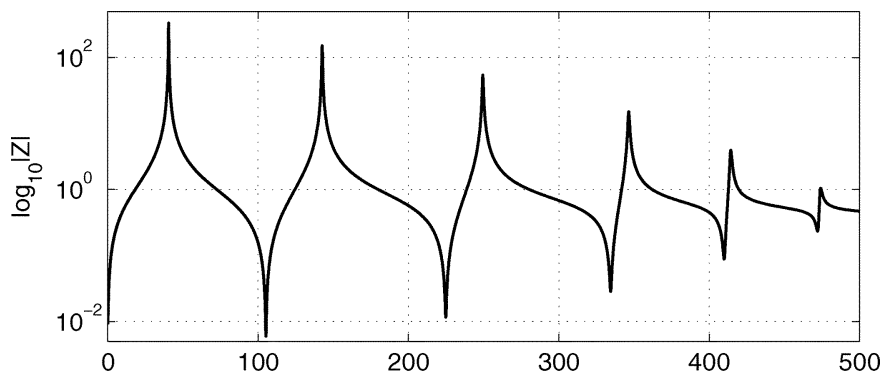

(a)

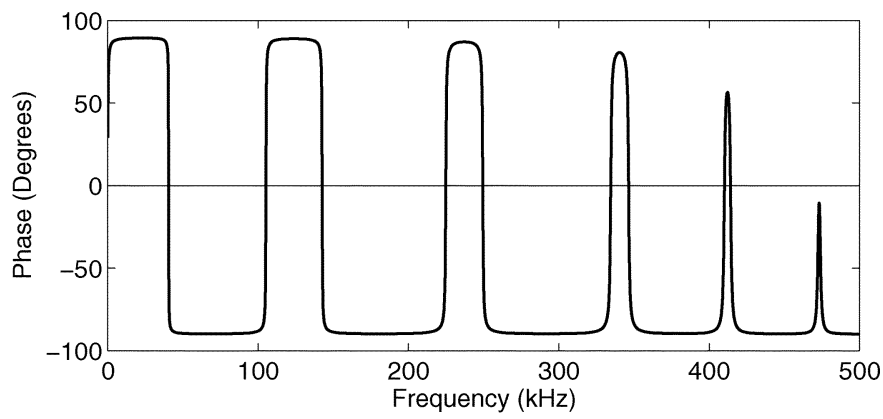

(b)

Fig. 4. Computed driving-point impedance of the synthesized circuit in Fig. 3. (a) Magnitude. (b) Phase.

in Table III, by which inductance matrix $\left[L_{\text {ref }}\right]$ can be constructed. The equivalent inductance $L_{\mathrm{eq}}$ and natural frequencies are presented in Table I.

The 6-section synthesized circuit characterized by the matrices $\left[R_{\mathrm{ref}}\right],\left[K_{\mathrm{ref}}\right]$, and $\left[L_{\mathrm{ref}}\right]$ is shown in Fig. 3. Its frequencyresponse plots are presented in Fig. 4 in which all of the wellpronounced natural frequencies of the model winding are seen.

2) Synthesizing Fresh Circuits After Introducing Changes: The ability of the proposed method to correctly locate the position and the extent of the change introduced in the model winding is demonstrated by means of the following case studies which are divided into three categories, each of them with increasing degree of complexity. The changes made in the model winding were confined only to capacitive changes, and due to practical difficulties in the available model winding, desired inductive changes could not be made.

\section{1) Changes pertaining to one tap}

In this group, changes are introduced in the model winding at a single tap (which can occur at any position
TABLE IV

MEAsured ANd Estimated Frequencies After Changes

\begin{tabular}{|c|c|c|c|c|}
\hline \multirow[t]{2}{*}{ Case } & \multicolumn{2}{|c|}{$\begin{array}{l}\text { Measured with } \\
\text { Model winding }\end{array}$} & \multicolumn{2}{|c|}{$\begin{array}{l}\text { Estimated using } \\
\text { Synthesized circuit }\end{array}$} \\
\hline & $\operatorname{senf}(\mathrm{kHz})$ & $\operatorname{ocnf}(\mathrm{kHz})$ & $\operatorname{senf}(\mathrm{kHz})$ & ocnf $(\mathrm{kHz})$ \\
\hline \multirow[t]{7}{*}{$\overline{X_{1}}$} & 104.97 & 38.80 & 105.19 & 39.00 \\
\hline & 224.80 & 138.90 & 224.81 & 138.33 \\
\hline & 336.15 & 247.00 & 334.47 & 245.20 \\
\hline & & 343.54 & 410.02 & 344.02 \\
\hline & & & 472.45 & 413.35 \\
\hline & & & & 473.49 \\
\hline & $C_{g}^{\prime \prime}(1,0):$ & $28 \rightarrow 0.5$ & $C_{g}(1,0): 0$ & $67 \rightarrow 0.65$ \\
\hline \multirow[t]{7}{*}{$X_{2}$} & 97.95 & 39 & 98.76 & 39.28 \\
\hline & 224.68 & 138.2 & 224.81 & 137.83 \\
\hline & 327.1 & 250.6 & 325.45 & 248.76 \\
\hline & & 340.2 & 410.02 & 337.96 \\
\hline & & & 465.18 & 414.15 \\
\hline & & & & 466.95 \\
\hline & $C_{q}^{\prime \prime}(6,0):$ & $56 \rightarrow 1.06$ & $C_{g}(4,0): 0$ & $33 \rightarrow 1.35$ \\
\hline \multirow[t]{7}{*}{$\overline{X_{3}}$} & 105.5 & 40.30 & 106.10 & 40.55 \\
\hline & 227.9 & 144.30 & 229.40 & 144.17 \\
\hline & 343.7 & 254.80 & 341.83 & 254.39 \\
\hline & & 354.89 & 416.58 & 353.36 \\
\hline & & & 475.35 & 420.30 \\
\hline & & & & 476.50 \\
\hline & $C_{g}^{\prime}(10,0)$ & $.56 \rightarrow 0.1$ & $C_{g}(6,0): 0$ & $33 \rightarrow 0.67$ \\
\hline \multirow[t]{8}{*}{$\overline{X_{4}}$} & 104.33 & 37.20 & 103.79 & 37.49 \\
\hline & 221.26 & 138.30 & 217.93 & 139.35 \\
\hline & 327.4 & 249.43 & 324.98 & 247.19 \\
\hline & & 346.20 & 403.81 & 340.25 \\
\hline & & & 470.14 & 408.29 \\
\hline & & & & 471.44 \\
\hline & $C_{g}^{\prime}(2,0):$ & $6 \rightarrow 1.06$ & $C_{g}(1,0): 0$ & $167 \rightarrow 0.667$ \\
\hline & & & $C_{g}(2,0): 0$ & $33 \rightarrow 1.333$ \\
\hline \multirow[t]{9}{*}{$Y_{1}$} & 101.3 & 38.4 & 101.52 & 39.15 \\
\hline & 216.7 & 144.6 & 219.54 & 143.68 \\
\hline & 331 & 246.8 & 337.52 & 244.74 \\
\hline & & 341 & 414.08 & 346.82 \\
\hline & & & 472.73 & 417.71 \\
\hline & & & & 473.75 \\
\hline & $C_{s}^{\prime}(3,4):$ & $\rightarrow 0.56$ & $C_{s}(2,3): 0$ & $\rightarrow 0.5$ \\
\hline & $C_{g}^{\prime}(4,0):$ & $56 \rightarrow 1$ & $C_{g}(3,0): 0$ & $33 \rightarrow 1.283$ \\
\hline & & & $C_{g}(2,0): 0$ & $33 \rightarrow 0.883$ \\
\hline \multirow[t]{10}{*}{$Y_{2}$} & 105.06 & 40.1 & 105.60 & 40.22 \\
\hline & 218.98 & 144.1 & 220.23 & 144.88 \\
\hline & 334.4 & 242.1 & 335.07 & 241.31 \\
\hline & & 353 & 405.87 & 350.90 \\
\hline & & & 473.61 & 407.70 \\
\hline & & & & 475.65 \\
\hline & $C_{s}^{\prime \prime}(5,6):$ & $\rightarrow 0.5$ & $C_{s}(3,4): 0$ & $\rightarrow 0.4$ \\
\hline & $C_{s}^{\prime}(6,7):$ & & $C_{s}(4,5): 0$ & $\rightarrow 0.9$ \\
\hline & & & $C_{g}(3,0): 0$ & $33 \rightarrow 1.033$ \\
\hline & & & $C_{g}(4,0): 0$ & $33 \rightarrow 0.833$ \\
\hline \multirow[t]{11}{*}{$Y_{3}$} & 97.93 & 38.9 & 98.37 & 38.98 \\
\hline & 218.97 & 138.9 & 218.18 & 138.73 \\
\hline & 324.7 & 240.9 & 325.82 & 238.98 \\
\hline & & 345.2 & 403.90 & 342.13 \\
\hline & & & 464.98 & 405.72 \\
\hline & & & & 467.67 \\
\hline & $C_{s}^{\prime}(5,6):$ & 0.5 & $C_{s}(3,4): 0$ & $\rightarrow 0.4$ \\
\hline & $C_{s}^{i}(6,7):$ & $\rightarrow 2$ & $C_{s}(4,5): 0$ & $\rightarrow 0.9$ \\
\hline & $C_{g}^{\prime}(6,0):$ & $56 \rightarrow 1.06$ & $C_{g}(4,0): 0$ & $33 \rightarrow 1.233$ \\
\hline & & & $C_{g}(3,0): 0$ & $33 \rightarrow 1.033$ \\
\hline & & & $C_{g}(5,0): 0$ & $33 \rightarrow 1.033$ \\
\hline \multirow[t]{8}{*}{$\overline{Z_{1}}$} & 110 & 38.8 & 109.51 & 39.01 \\
\hline & 224.9 & 141 & 224.81 & 140.16 \\
\hline & & 245.3 & 341.17 & 244.03 \\
\hline & & & 410.02 & 349.73 \\
\hline & & & 479.10 & 413.11 \\
\hline & & & & 479.81 \\
\hline & $C_{g}^{\prime \prime}(1,0):$ & $28 \rightarrow 0.56$ & $C_{g}(1,0): 0$ & $167 \rightarrow 0.73$ \\
\hline & $C_{g}^{\prime}(6,0):$ & $56 \rightarrow 0.28$ & $C_{g}(4,0): 0$ & $33 \rightarrow 0.69$ \\
\hline
\end{tabular}

(ii) $A \rightarrow B$ implies a change from $A$ to $B$

along the winding), namely $X_{1}$ is at the line end, $X_{2}$ is at the middle, while $X_{3}$ and $X_{4}$ are close to the neutral end and line end, respectively.

Results pertaining to Case $X_{1}$ are presented below. Here, the shunt capacitance at the line end is increased from the reference value of $0.28 \mathrm{nF}$ to $0.5 \mathrm{nF}$, that is, $\Delta C_{g}^{\prime}(1,0)=+0.22 \mathrm{nF}$. The scnfs and ocnfs of the model winding (after the changes are made) are measured and are presented in Table IV.

Corresponding to the changed set of natural frequencies, a new equivalent circuit of six sections (topology 
remaining unchanged) is synthesized, as per procedure in Section V. The scnfs and ocnfs of the synthesized circuit are also presented in Table IV. The resulting matrices $[\widehat{K}]$ and $[\widehat{L}]$ of the synthesized circuit are

$$
\begin{aligned}
{[\widehat{L}] } & =\left[L_{\text {ref }}\right] \\
\widehat{K}(i, j) & = \begin{cases}1.25 \mathrm{nF}, & i=j=1 \\
K_{\text {ref }}(i, j), & \text { otherwise. }\end{cases}
\end{aligned}
$$

The above signifies that only the shunt capacitance at the line end $\left(C_{g}(1,0)\right)$ has changed, thus indicating that the change was made at the line end in the model winding and the quantum of change is estimated to be

$\Delta C_{g}(1,0)=\widehat{K}(1,1)-K_{\mathrm{ref}}(1,1)=+0.183 \mathrm{nF}$.

(Note: $K_{\text {ref }}(1,1)=\left(C_{g} / 2\right)+C_{s}=0.467+0.6=$ $1.067 \mathrm{nF})$.

From the above, it is found that the location at which the change was introduced in the model winding has been correctly identified and also the estimated change is nearly equal to the change introduced.

Following are the other case studies wherein changes are associated with one tap alone.

- In Case $X_{2}$, the shunt capacitance at the middle (i.e., TAP 6) of the winding was increased from $0.56 \mathrm{nF}$ to $1.06 \mathrm{nF}$ and it gets reflected as an increase of the shunt capacitance at the middle node (i.e., NODE 4) in the synthesized circuit from $0.933 \mathrm{nF}$ to $1.35 \mathrm{nF}$.

- In Case $X_{3}$, the shunt capacitance close to the neutral end (i.e., TAP 10) of the winding decreased from $0.56 \mathrm{nF}$ to $0.1 \mathrm{nF}$ and this was identified as a decrease in the value of the shunt capacitance close to the neutral end (i.e., NODE 6) in the synthesized circuit from $0.933 \mathrm{nF}$ to $0.67 \mathrm{nF}$. In cases $X_{2}$ and $X_{3}$, it was observed that a discrete change introduced in the model winding is mapped to a discrete node in the synthesized circuit.

- In Case $X_{4}$, although only the shunt capacitance at TAP 2 alone is changed (in the model winding), the change gets reflected at two nodes (1 and 2) in the synthesized circuit and the reason for this will be explained later.

The corresponding measured and estimated natural frequencies for all of these cases are presented in Table IV.

\section{2) Changes pertaining to more than one tap}

In these cases, the introduced changes affect more than one tap in the model winding. In Case $Y_{1}$, series capacitance between TAPS 3 and 4 decreased from $1 \mathrm{nF}$ to $0.56 \mathrm{nF}$ and simultaneously, the shunt capacitance at TAP 4 increased from $0.56 \mathrm{nF}$ to $1 \mathrm{nF}$. In Case $Y_{2}$, series capacitances $C_{s}^{\prime}(5,6)$ and $C_{s}^{\prime}(6,7)$ were simultaneously decreased and increased from the reference value (that is, $0.6 \mathrm{nF}$ ) to $0.5 \mathrm{nF}$ and $2 \mathrm{nF}$, respectively. It was desirable to study the combined effect of two different cases. For this purpose, in Case $Y_{3}$, all of the changes corresponding to Cases $X_{2}$ and $Y_{2}$ were simultaneously introduced in the model winding (see Table IV).
The ocnfs and scnfs corresponding to Cases $Y_{1}, Y_{2}$ and $Y_{3}$ were measured and are presented in Table IV. Then, equivalent circuits are synthesized for all of the cases as explained earlier. The resulting natural frequencies of the synthesized circuits are also computed and presented in Table IV. A comparison of these synthesized circuits with the reference circuit reveals the position and the extent of the changes made and these are also mentioned in Table IV against each case.

\section{3) Changes to physically separated taps}

In reality, mechanical deformations often result in damages that affect different parts of the winding. Hence, it is interesting to simulate such a situation to the extent possible. With this in mind, in Case $Z_{1}$, capacitances at TAPS 1 (line end) and 6 (middle) were simultaneously increased and decreased, respectively, by the same margin. As a result, the net change in $C_{\mathrm{g} \text {,eff was }}$ zero. The synthesized circuit for this case was obtained following the same procedure and the changes were reflected at NODES 1 (line end) and 4 (middle) in the synthesized circuit.

The following salient points emerge from the above results and are discussed below.

- It is observed that all of the natural frequencies of the synthesized circuits are found to be well within 1-2\% of the corresponding measured values. This aspect indicates the agreement of the terminal characteristics of the model winding and synthesized circuit.

- In all of the case studies by observing the changed elements in the synthesized circuits, the actual position of the changes introduced in the model winding were successfully traced. From these results, it emerges that a correlation exists between the model winding and the synthesized circuit. This is schematically shown in Fig. 5 along with the mapping results, which link changes introduced at taps in the model winding to the nodes in the synthesized circuit. Thus, it emerges that localization of the changes introduced in the model winding is possible.

- Percentage of error between the natural frequencies of the synthesized circuit and the model winding was in the range of $1-2 \%$ in all of the above cases. Efforts to further reduce this error did not result in any further improvement, which indicates that this error cannot arbitrarily be reduced. This is because of the following reasons.

1) Accurate identification of the current minima (ocnfs) during the measurements is significantly affected by voltage sensitivity and finite resolution of digital oscilloscope (despite employing averaging techniques), current probe sensitivity, and harmonic distortion/background noise level associated with the function generator.

2) In Cases $X_{4}, Y_{1}, Y_{2}$, and $Y_{3}$, it was observed that the discrete changes introduced in the model winding were not correspondingly reflected by the same number of discrete changes in the synthesized circuit. This aspect should not be construed as an error and can be explained using Fig. 5. It can be observed that all taps in the winding are not getting 


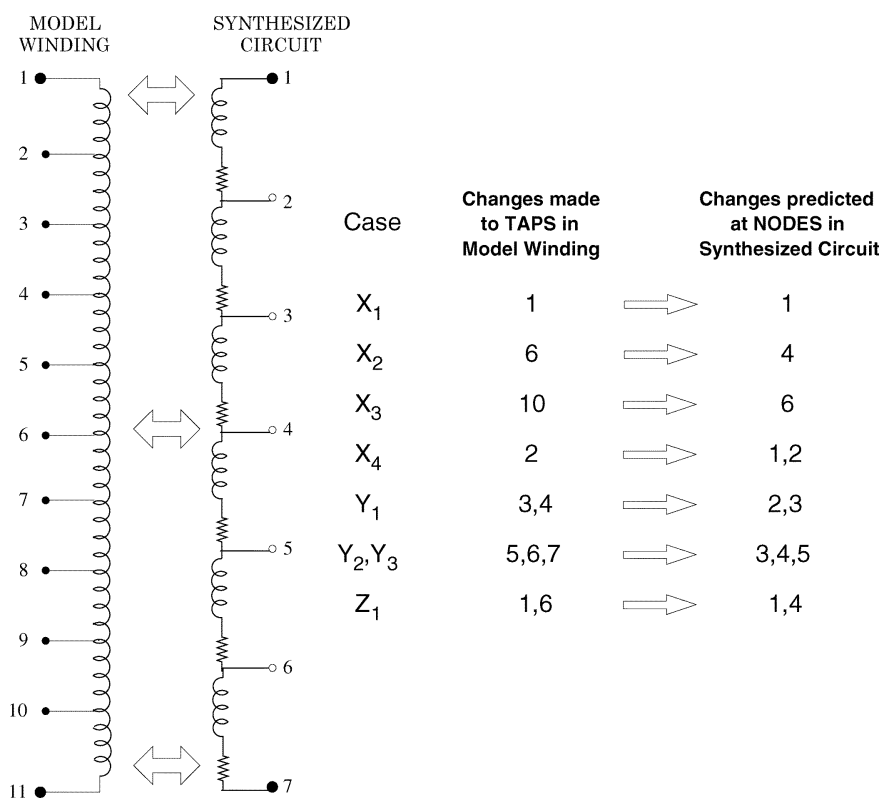

Fig. 5. Mapping of taps in model winding to nodes in the synthesized circuit ( $C_{s}$ and $C_{g}$ exist, but are not shown).

mapped on to discrete nodes in the synthesized circuit, except TAPS 1,6 , and 11 which map to NODES 1,4 , and 7, respectively. Therefore, when changes involve taps other than 1,6, and 11, a slight spread in the predicted nodes is observed, which is limited to one node on either side.

- In Case $Y_{2}$, it is seen that a variation of both the series capacitances linked with TAP 6 (i.e., $C_{s}^{\prime}(5,6)$ and $C_{s}^{\prime}(6,7)$ ) in the model winding results in a change in the series capacitances linked with NODE 4 (i.e., $C_{s}(3,4)$ and $C_{s}(4,5)$ ) in the synthesized circuit. Further, it is found that the nature of the changes (increase/decrease) introduced in the model winding are also correspondingly reflected in the synthesized circuit.

- It is very interesting to see the results pertinent to Case $Y_{3}$, wherein the changes corresponding to Cases $X_{2}$ and $Y_{2}$ were simultaneously introduced in the model winding and the changes estimated in the circuit are almost the same as the combined effects of the individual cases.

- In Case $Z_{1}$, despite no net change in the value of $C_{\mathrm{g}, \mathrm{eff}}$ (refer Table IV), the method was able to correctly identify the changes made in the model winding. This demonstrates the ability of the method to identify multiple changes occurring at physically different locations along the model winding.

\section{B. Case Studies With Interleaved Windings}

The case studies discussed until now pertain to a continuous-disc winding. As the behavior of an interleaved winding is known to be peculiar [20], it becomes important to examine whether the proposed method has any limitation, with regard to the type of the winding. Hence, case studies were repeated for both fully and partially interleaved winding representations. The results were encouraging (for details, see [21]). (a)

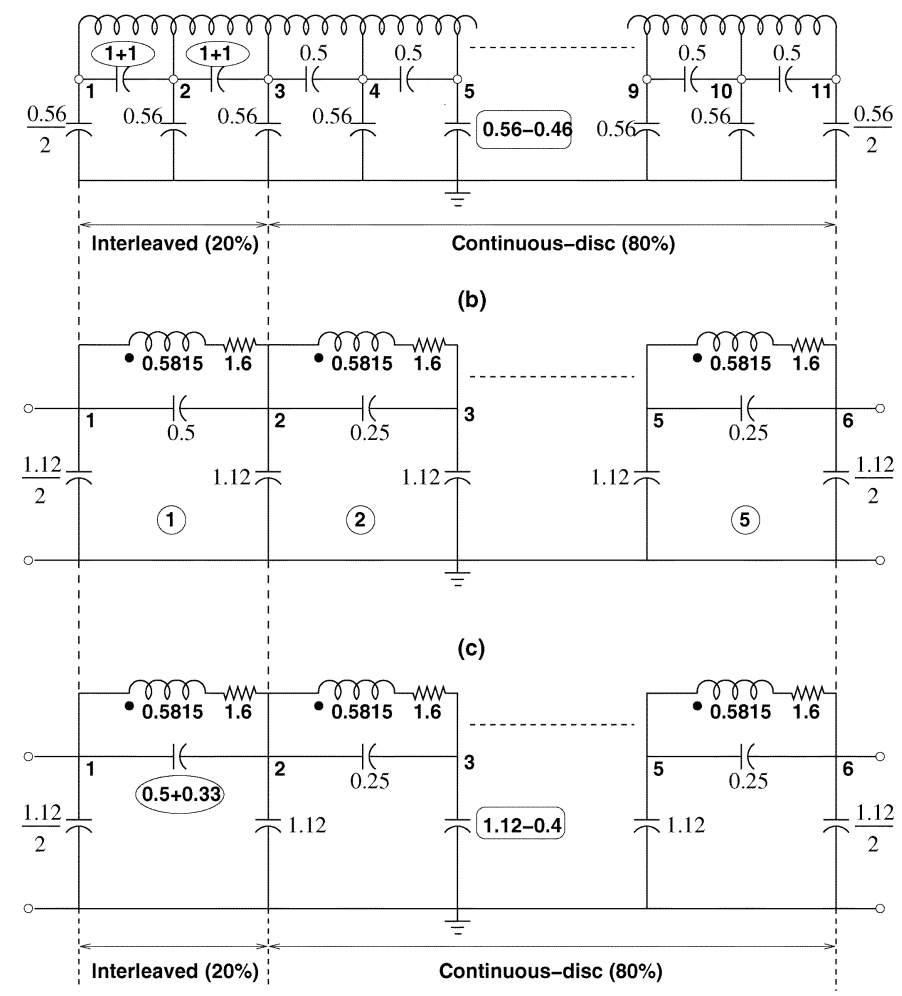

Fig. 6. (a) $20 \%$ partially interleaved winding (encircled values show the position of the changes). (b) Synthesized 5-section reference circuit. (c) Synthesized circuit showing predicted changes (encircled).

One interesting case study pertaining to a partially interleaved winding is presented here. In a partially interleaved winding, voltage distribution constant $(\alpha)$ would be different for different parts of the winding. To represent such a winding (with 20\% interleaving), capacitances are connected as shown in Fig. 6(a) such that:

- shunt capacitance to ground $C_{\mathrm{g}, \mathrm{eff}}=5.6 \mathrm{nF}$;

- $20 \%$ interleaved at line end with $\alpha=1.4967$ and the remaining $80 \%$ is continuous disc with $\alpha=8.4664$.

Natural frequencies of this model winding are measured and the scaling factor $\beta_{1}$ was determined. Using condition (8), $N$ was found to be 5 . Since the percentage of interleaving is $20 \%$, an attempt was made to synthesize a 5 -section equivalent circuit in the ratio of 1:4.

(Note: If $N=5$ does not satisfy (8), then $N=10$ needs to be considered as this is the next permissible value that could satisfy $20 \%$ interleaving criteria.)

With this model winding, case studies similar to those presented earlier were repeated. In all of them, it was observed that the changes estimated by the synthesized circuit corresponded to the changes introduced in the model winding. One sample result is presented in Fig. 6 .

Fig. 6(a) represents a 20\% partially interleaved model winding. The synthesized 5-section reference circuit is shown in Fig. 6(b). In Fig. 6(a), $C_{g}$ at TAP 5 was decreased from $0.56 \mathrm{nF}$ to $0.1 \mathrm{nF}$ and $C_{s}$ between TAPS $1-2$ and 2-3 were increased from $1 \mathrm{nF}$ to $2 \mathrm{nF}$. The series capacitance change is only $+0.5 \mathrm{nF}$, which is seen as a $0.33-\mathrm{nF}$ change in the synthesized circuit. The shunt capacitance change in the model 
winding is $-0.46 \mathrm{nF}$ and is identified as a change of $-0.4 \mathrm{nF}$ in the synthesized circuit. Thus, the changes introduced in the model winding were correctly identified in the synthesized circuit shown in Fig. 6(c) (The encircled values show the detected changes). Thus, it has been shown that the method works equally well for both types of winding representations.

\section{FUTURE SCOPE}

Experimental studies were presented to demonstrate the capability of the proposed method to localize specific changes introduced in a model winding, based on terminal measurements. Notwithstanding that only a model winding was considered, an assessment of the localization results predicted in the various case studies indicated that the method seems to possess some potential and, hence, merits further consideration. So future explorations must examine the following issues.

1) Initially, the method (considering model winding) needs to be examined when changes in inductances are made. This would require minor modifications pertaining to the estimation of self and mutual inductances.

2) In practice, resistance of the winding increases due to skin effect. This results in lower magnitudes of peaks in the FRA plot. In addition, the phase transitions at ocnf and scnf are not sharp. This aspect can be modelled by including frequency-dependent resistance. Resistances at different frequencies can be estimated based on the magnitude changes in FRA plots.

3) The next step would be to implement the proposed method on an actual transformer winding. For this, mechanical deformations have to be intentionally made at different locations, and the outcome of the method needs to be examined.

4) If the performance is acceptable, then it would be worthwhile to consider the presence of neighboring windings and core, and their influences ascertained.

The above are additional reasons as to why the study was restricted to only a model winding. Therefore, it can be visualized that a solution suited for an actual transformer winding will require more work and effort.

\section{CONCLUSION}

The ability to locate portions of a transformer winding which are physically deformed, based on terminal measurements, still remains as a very challenging problem. In this regard, this paper has demonstrated how discrete changes made in a model winding could be correctly located (based on terminal measurements alone) and, therefore, can be considered as a small progress. The proposed method employs an iterative circuit synthesis procedure and constructs a ladder network, based on information about short-circuit/open-circuit natural frequencies and some terminal data. In this manner, it establishes a mapping between the model winding and the synthesized circuit, and on this count can be considered a novel contribution. Finally, it is believed that these findings could be of assistance in locating and quantifying mechanical deformations in actual transformer windings.

\section{REFERENCES}

[1] W. Lech and L. Tyminski, "Detecting transformer winding damage by the low voltage impulse method," Elect. Rev., vol. 179, no. 21, pp. 768-772, Nov. 1966.
[2] E. P. Dick and C. C. Erven, "Transformer diagnostic testing by frequency response analysis," IEEE Trans. Power App. Syst., vol. PAS-97, no. 6, pp. 2144-2153, Nov./Dec. 1978.

[3] R. Malewski and B. Poulin, "Impulse testing of power transformers using the transfer function method," IEEE Trans. Power Del., vol. 3, no. 2, pp. 476-489, Apr. 1988.

[4] T. Leibfried and K. Feser, "Monitoring of power transformers using the transfer function method," IEEE Trans. Power Del., vol. 14, no. 4, pp. 1333-1341, Oct. 1999.

[5] K. Okuyama, "A numerical analysis of impulse voltage distribution in transformer windings," Elect. Eng. Jpn., vol. 87, no. 1, pp. 80-88, 1967.

[6] A. Miki, T. Hosoya, and K. Okuyama, "A calculation method for impulse voltage distribution and transferred voltage in transformer windings," IEEE Trans. Power App. Syst., vol. PAS-97, no. 3, pp. 930-939, May/Jun. 1978.

[7] F. de Leon and A. Semlyen, "Efficient calculation of elementary parameters of transformers," IEEE Trans. Power Del., vol. 7, no. 1, pp. 376-383, Jan. 1992.

[8] A. Oguz Soysal, "A method for wide frequency range modeling of power transformers and rotating machines," IEEE Trans. Power Del., vol. 8, no. 4, pp. 1802-1808, Oct. 1993.

[9] R. C. Degeneff, "A general method for determining resonances in transformer windings," IEEE Trans. Power App. Syst., vol. PAS-96, no. 2, pp. 423-430, Mar./Apr. 1977.

[10] P. T. M. Vaessen, "Transformer model for high frequencies," IEEE Trans. Power Del., vol. 3, no. 4, pp. 1761-1768, Oct. 1988.

[11] A. Morched, L. Martf, and J. Ottevangers, "A high frequency transformer model for the EMTP," IEEE Trans. Power Del., vol. 8, no. 3, pp. 1615-1626, Jul. 1993.

[12] A. Keyhani, H. Tsai, and A. Abur, "Maximum likelihood estimation of high frequency machine and transformer winding parameters," IEEE Trans. Power Del., vol. 5, no. 1, pp. 212-219, Jan. 1990.

[13] D. M. Sofian, Z. D. Wang, and P. Jarman, "Interpretation of transformer FRA measurement results using winding equivalent circuit modeling technique," in Proc. Conf. Electrical Insulation and Dielectric Phenomena, Nashville, TN, Oct. 16-19, 2005, pp. 613-616.

[14] E. C. Levy, "Complex curve fitting," IRE Trans. Autom. Control, vol. AC-4, pp. 37-44, May 1959.

[15] A. H. Whitfield, "Transfer function synthesis using frequency response data," Int. J. Control, vol. 43, no. 5, pp. 1413-1426, 1986.

[16] B. Gustavsen and A. Semlyen, "Rational approximation of frequency domain responses by vector fitting," IEEE Trans. Power Del., vol. 14, no. 3, pp. 1052-1061, Jul. 1999.

[17] J. Vlach, Computerized Approximation and Synthesis of Linear Networks. New York: Wiley, 1969.

[18] E. A. Guillemin, Synthesis of Passive Networks. New York: Wiley, 1962.

[19] P. A. Abetti and F. J. Maginniss, "Natural frequencies of coils and windings determined by equivalent circuit," AIEE Trans., vol. 72, pt. III, pp. 495-504, Jun. 1953.

[20] L. Satish and A. Jain, "Structure of transfer function of transformers with special reference to interleaved windings," IEEE Trans. Power Del., vol. 17, no. 3, pp. 754-760, Jul. 2002.

[21] K. Ragavan, "An efficient solution to generalized model of a transformer winding and localization of discrete changes based on measurements," Ph.D. dissertation, High Voltage Lab., Dept. Elect. Eng., Indian Inst. Sci., Bangalore, India, 2006.

K. Ragavan was born in India in 1976. He received the B.Tech. degree from Pondicherry Engineering College, Pondicherry, India, in 1997, the M.E. degree from Anna University, Chennai, India, in 2002, and the Ph.D. degree from the Indian Institute of Science, Bangalore, India, in 2006.

He is currently working in GE India Technology Centre Pvt. Ltd., Bangalore.

L. Satish (SM'02) was born in 1964. He received the Ph.D. degree from the Indian Institute of Science (IISc), Bangalore, India, in 1993.

He was a Postdoctoral Fellow with ETH, Zurich, Switzerland, from 1993 to 1995. He joined the Department of High Voltage Engineering, IISc, in 1995, where he is currently an Associate Professor. His research interests include application of signal processing to high-voltage impulse testing, diagnostics, condition monitoring, PD measurements, pattern recognition, and analog-to-digital converter (ADC) testing. He was a Visiting Researcher at H.V. Institute, H.U.T, Finland, in 1998.

Dr. Satish is a member of CIGRE Working Group D1-33. 